PROMOTING MULTIDISCIPLINARY RELATIONSHIPS

\title{
PROMOTING MULTIDISCIPLINARY RELATIONSHIPS: A PRAGMATIC FRAMEWORK FOR HELPING SERVICE PROVIDERS TO WORK COLLABORATIVELY
}

\author{
LYNNE M. ROBINSON \\ Dalhousie University
}

\begin{abstract}
Multidisciplinary teamwork is increasingly considered the ideal way to provide mental health services. This is especially the case when working with children or families. However, there is little easy-to-use information available to help practicing professionals recognize and overcome barriers to working in multidisciplinary settings. There are even fewer resources intended for use across disiplines. This paper offers a practical approach to help mental health professionals identify barriers to teamwork and create solutions to those barriers.
\end{abstract}

\section{INTRODUCTION}

More and more mental health service organizations are becoming involved in multidisciplinary work. ${ }^{1}$ In work with children and families, for example, there has been for some years now a growing consensus that services provided by a range of disciplines and agencies should be integrated into a coherent set of interventions (APA, 1994; Dosser, Handron, McCammon, Powell, \& Spencer, 2001). Yet extending that consensus to work with seriously mentally ill persons poses special challenges (Johnson, Wistow, Schulz, \& Hardy, 2003). Indeed, there remains a real lack of broadly applicable, pragmatic information available on the problems that develop among providers within multidisciplinary settings and, even more importantly, of solutions to those problems. Most providers are now trained in dealing with the multicultural issues of their clients; however, very few of them are trained to deal with the "multicultural" issues of their colleagues (Haley et al., 2004).

Divisions within a team-whether that team is permanent or has been created around one issue or family - can cause problems far beyond the initial team members. If animosity develops between two individuals, it is likely to fester and, worse still, to create divisions in the provider community as people line up on one side or the other. This issue is significant in the training of new practitioners in all the fields involved in providing mental health-related services. As Seaburn suggests, "It is impossible to provide good collaborative care if the providers involved do not know how to work together" (2001, p. 48). Further, "a culture of collaboration does not just happen. It must be formed by many hands” (Seaburn, Lorenz, Gunn, \& Gawinski, 1996, p. 23).

In this paper, I offer a review of the literature on collaborative practice (including theories about permanent teams, training for multidisciplinary practice, and reports on collaborative practices) and identify some key features of well- 
functioning groups, some commonly identified barriers to collaboration, and some key skills for working in multidisciplinary ways that are useful in helping practitioners to collaborate well. Further, I offer a usable and pragmatic approach for thinking about how to create and maintain working relationships through recognizing barriers and solving interpersonal problems in multidisciplinary work environments.

\section{LITERATURE REVIEW}

There is a growing body of information available on collaborative practice which reflects upon as many as five different types of interdisciplinary teams (Drinka \& Clarke, 2000). Much of the literature is based on primary care settings (e.g., Bray, Enright, \& Rogers, 1997). Some works define or assume teams to be relatively stable and enduring (e.g., Hackman, 2004; Lambert, 2002); others describe how organizations or groups can create and support collaboration (e.g., Drinka \& Clark, 2000; Heinemann \& Zeiss, 2002a); while others focus on collaboration with families (e.g, Seaburn et al., 1996). Unfortunately, most of the material presents a set of desired characteristics for multidisciplinary teams but provides relatively little pragmatic information about how to achieve those characteristics.

The existing literature focuses on many different aspects of teamwork, from designating the roles of each discipline (Liberman, Hilty, Drake, \& Tsang, 2001) to describing the phases of team development (Drinka \& Clark, 2000) to identifying tools for assessing the functioning of teams (Heinemann \& Zeiss, 2002b). Thus, for workers in the field who have limited time to read, collate, and reflect upon new materials, the myriad details provided in the literature are almost impossible to operationalize. Further, even though Waxman, Weist, and Best suggest that "it can take 3-5 years for a working group of team members to form a cohesive partnership" (1999, p. 245), much multidisciplinary work takes place within loosely structured teams created to serve a particular client or temporary need. In such circumstances, the gradual development of team processes often is not possible.

Some authors have identified different levels of analysis relevant to interdisciplinary work. Drinka and Clark (2000) refer to individual, intra-team, and organizational issues. Hargrove and Keller (1997) identify structural factors (external forces) and personal factors (consisting of internal characteristics of key workers in organizations). These two models are not interchangeable since, for example, Drinka and Clark assign elements to the "individual" level that do not fit within the "personal” level of Hargrove and Keller. In general, however, the efficacy of a multidiciplinary team can be impacted by factors which operate at the organizational, team, and individual levels (Heinemann, 2002; Heinemann \& Zeiss, 2002a). Multidisciplinary work is strongest when there is explicit support for group processes at all three levels. In practice, however, such support often is not available, and individual practitioners must learn to negotiate group processes on their own. Accordingly, Johnson and colleagues (2003) note that integrating organizational structures will not create true collaboration unless there is a focus on the individuals who are expected to collaborate.

Many authors note similar features of well-functioning interdisciplinary teams. A key organizational feature is good administrative support (Liberman et al., 2001). At the team level, leadership and appropriate power structures often are identified (Drinka \& Clark, 2000; Liberman et al., 2001; Nichols, DeFriese, \& Malone, 2002; Sanders et al., 1999). For individuals, important attributes for a well-functioning team include: (a) good communication skills (Felker et al., 2004; Nichols et al., 2002; 
Sanders et al., 1999), which includes the use of accessible language (Caplan \& Caplan, 1993; Haley et al., 2004; Seaburn et al., 1996); (b) strong interpersonal relationships; and (c) respect among team members (Harmon, Braillier, \& Brown, 2002; Sanders et al., 1999; Weist et al., 2001). There are many other, less commonly reported features of well-functioning teams that have been identified at the organizational or team level, such as the integration of members with needed expertise into team processes and accountability of team members (Liberman et al., 2001).

Although the features of well-functioning teams and the skills of productive team members are similar, it is important to differentiate them. It is possible to facilitate positive team features both through appropriate structuring of the collaboration context and through training team members in needed skills (Lewandowski \& GlenMaye, 2002). It also may be helpful to select team members with skills that reflect desired team features. Most authors agree on how important it is for all providers to treat one another with respect and to value the different types of input that each profession brings to the team (Boone, Minore, Katt, \& Kinch, 1997; Sanders et al., 1999). It can be useful to consider these traits not only as aspects of the team process but also as skills in which the individual can be trained. There is general agreement in the literature that individual service providers need good communication skills (Nichols et al., 2002) and strong conflict resolution skills (Fatout \& Rose, 1995; Nichols et al., 2002). They also need to be flexible, creative, and open to learning from others (Nichols et al., 2002) and to have knowledge of other professions (Haley et al., 2004). Unfortunately, while some of the people providing mental health care have these abilities, others do not.

Clearly, there is a need for a brief, pragmatic framework that mental health care providers can use to ensure well-functioning multidisciplinary teams. As Patterson (2001) points out, training plays a key role in developing the capacity to provide collaborative care. Joseph (2001) concurs. She describes the "hunger" that she sees in her students-medical residents in search of concrete suggestions rather than abstract principles. I believe, as do many others (Seaburn et al., 1996; Davis, 2001; Felker et al., 2004; Sanders, Brockway, Ellis, Cotton, \& Bredin, 1999; Weist, Lowie, Flaherty, \& Pruitt, 2001; Caplan \& Caplan, 1993; Waxman et al., 1999), that such hunger can best be satisfied by providing training at the level of interpersonal relationships. If, as Liberman and colleagues suggest, "the concept of multidisciplinary work is a vision in search of practical tools" (2001, p. 1335), then those tools will be focused on promoting positive interpersonal relationships and solving problems between team members.

\section{PROMOTING MULTIDISCIPLINARY RELATIONS}

Perhaps the single most commonly identified barrier to effective multidisciplinary teams is the distinctiveness which each discipline brings to the collaboration (Bray et al., 1997; Drinka \& Clark, 2000; Fatout \& Rose, 1995; Ruddy \& Schroeder, 2004; Waxman et al., 1999). The differences between disciplines are so marked that Seaburn and colleagues (1996) describe the disciplines as belonging to different professional cultures. The nature of the differences have been variously described as dealing with: (a) unique approaches to language, treatment stance (i.e., directive vs. collaborative), time-management, and overall orientation (biomedical or psychosocial) (McDaniel, Campbell, \& Seaburn, 1990); (b) issues of client expectations and standards of confidentiality (Waxman et al., 1999); (c) conflicts of interest between 
staff and ways in which perceptions of other workers become distorted (because of culture, stereotyping, past experiences, etc.) (Caplan \& Caplan, 1993); (d) political struggles, such as "turf" battles and power differences (Caplan \& Caplan 1993; Liberman et al., 2001; Sanders et al., 1999; Waxman et al., 1999); and (e) lack of knowledge or acceptance of the roles of various disciplines (Weist et al., 2001).

Organizational issues such as differences between organizations and staff turnover have been noted by Freeth (2001) while Caplan and Caplan discuss the ways that poorly functioning organizations affect collaboration (1993). Lack of time for collaborative processes is cited by a number of authors (e.g., Johnson et al., 2003; Nichols et al., 2002). Many of these factors have their effect through organizational policies or through the practicalities of how the organization functions.

Despite the growing stream of writings on collaboration, practitioners from a variety of fields tend to focus heavily on their relationships with their clients, ignoring relationships with their colleagues. We, as care providers, tend to expect other practitioners to provide perfect services in the manner of the discipline that we are trained in. When this doesn't happen conflict can arise. It is important to apply the same sets of professional skills that we use with clients and their families to working with the interdisciplinary team itself. Understanding and managing interprofessional relationships is as crucial as understanding and managing practitioner-client relationships. This paper offers a usable and pragmatic approach for thinking about how to create and maintain working relationships through recognizing barriers and solving interpersonal problems in multidisciplinary work environments. The approach is suitable for a variety of disciplines. It is written in clear language without the jargon that so often impedes interdisciplinary communication and is in the form of an easy to remember mnemonic: the Promoting Multidisciplinary Relationships (PMR) system. It is intended to be used as a starting point for professionals in working groups to train themselves or each other. In order to help this process, illuminative examples are given, but those using this approach are encouraged to develop their own set of examples with solutions.

Most of us have learned to be self-reflective about our work with clients. It is just as important to be self-reflective about our professional relationships. I encourage readers to reflect on their own experiences of the ways in which these factors limit our ability to truly work together. In order to create an easy to teach and remember set of suggestions, the barriers, solutions, and the focus on the importance of the interdisciplinary relationships are summarized as the Promoting Multidisciplinary Relationships technique. The $P$ refers to the five barriers, the $M$ to the four solutions, and the $R$ to the importance of focusing on the relationships (See Table 1). Many authors identify good relationships as the most essential element of collabora-

\section{TABLE 1}

The PMR System: Barriers, Solutions and Relationship

\begin{tabular}{lll}
\hline Barriers (Ps) & Solutions (Ms) & Relationship (R) \\
\hline People & Make sense out of interactions & Focus on relationships \\
Professional cultures & Meet needs & \\
Policies & Mend fences & \\
Politics & Maintain relationships & \\
Practicalities & & \\
\hline
\end{tabular}


tion (Felker et al., 2004; Sanders et al., 1999; Seaburn et al., 1996; Weist et al., 2001) and thus the model emphasizes that element.

\section{Barriers to Collaborative Work: The Five Ps}

Based on the key findings in research on collaboration, and on my own experience of interdisciplinary teams, I suggest that difficulties generally come from what I have called, as a useful mnemonic, the five $P$ factors: people, professional cultures, policies, politics, and practicalities. The people factor refers to the reality that practitioners are also people, with all the baggage that each of us brings to any encounter. I consider that many of the key skills for collaboration as identified in the literature fall into this category. Such skills include the ability to communicate, to resolve conflict, and to be respectful of and open to other disciplines (e.g., Nichols et al., 2002). The professions factor refers to the culture that each profession has developed, including such things as language and accepted techniques. This factor is the one that has been most discussed in the literature as a barrier (e.g., Seaburn et al., 1996). As noted, many differences between professional cultures contribute to this factor. Policies refers to the various and often competing formal requirements of different services. Relatively few writers have explored the problems of incompatible policies, but Waxman and colleagues refer to one such type of policy as a barrier (1999). Politics refers both to the bigger, often covert, political agendas that most professions have and to the influence of larger political tides on agencies. Many authors note the importance of political struggle as a barrier (e.g., Caplan \& Caplan, 1993). Finally, practicalities refers to the realities of our daily working lives, as we struggle to meet the needs of our clients, of our own and other systems, and, last but not least, ourselves. Lack of time is perhaps the most important practicality, and is cited by a number of authors as a significant barrier (e.g., Johnson et al., 2003).

People

Each of us has our own way of working. We have our own personality styles. Some of us are more obsessive and concerned with structure, setting up policies and rules, and sticking to our mandates while others are more easygoing and concerned with going with the flow, feelings, and global understandings. We need both perspectives, but different personality types don't always understand each other.

We also don't tend to see the limitations on what others can find the time to do. We see that we are tired and needing to limit the new cases that we take on but we often aren't aware of the stresses others are going through — at least not until we take the time to get to know them. For example, in one program, workers funded from one agency knew that the workers from another agency had been understaffed for at least one year, yet some didn't recognize the devastating effect that this situation, coupled with a number of personal tragedies, had had on the other program. They could be critical of the failure of the other program to carry out needed services since they didn't have the close connections that would enable them to recognize the stresses.

Even professionals can have bad days and sometimes bad weeks, when their own lives become so stressed that their problems spill over into work. We need to try to be as accepting of other professionals as we are of our clients. Rather than blaming a colleague, it is important to step back and understand what might be affecting that person's work. Simply acknowledging the strain that colleagues are working under can free up emotional space to develop solutions. Better yet is asking colleagues to explain what they are struggling to deal with in their own work situations. 


\section{Professional cultures}

Each profession has its own way of understanding things and its own language. It is important to recognize that each culture has developed in response to the tasks of that profession. An example of an area that can be conflictual is in the protection of children. Social services workers sometimes think that health care workers don't do enough to help them protect children. Mental health workers may feel that complaints about abuse or neglect don't result in changes in the lives of children. The protective tasks of the professions are different and evolve from hard-won learning about what can and cannot be done within each role. One useful way to frame this discussion for team members is to recognize that everyone has a duty to protect children. Social services must investigate complaints, but health services must report any concerns to social services and be prepared to stand by their opinions.

When people work exclusively within their own cultures, they can go back and complain to their own group about their problems with "those other people," very often receiving substantial sympathy. This process can increase the sense of distrust between groups. There is a tendency for professionals who work with people to fall into the "power fallacy." Providers tend to either idealize what others can do or demonize them. On the one hand, it is common to imagine that the "other" has some magical ability to transform a child or family that we ourselves find very difficult to help. On the other hand, workers may feel real anger that another member of the team is "hurting" the client in some way.

When one works closely with an individual or group, one tends to know them well and empathize with them. There is a complementary tendency to blame the individual who is not worked with. This phenomenon is well known to anyone who works with families and it is important to understand the implications for working with larger groups. A therapist may blame a psychiatrist for "overmedicating" children and, similarly, the psychiatrist may feel that the therapist has been inadequate in helping a family manage the child's acting out problems.

There can be a lot of confusion when individuals trained in different paradigms work together. Use of specialized language can impede understanding of the work to be done and emphasize status differences. Just as when working with families, it is important to use terms that everyone can understand. This has the added benefit of making sure that you yourself understand what you are saying. Thorndike (1919) refers to the effect of using the same word for different meanings as the "jingle fallacy.” Kelley (1927) refers to using different words for the same meaning as the "jangle fallacy." And Block (1995) talks about such confusions as the "jingle jangle jungle” (all three cited in Hinde, 1999).

Language can also emphasize power differentials among members of the team. For example, there is often disagreement over what to call the people we serve. Are they clients, patients, or even customers? As a psychology graduate student, I felt devalued when a well-regarded physician began a seminar by announcing that psychologists would never be respected as long as we talked about clients rather than patients. Frequently, members of one profession feel that their way of working is not respected or understood by members of other professions. It can help to identify that the terms that each group uses are valid for the relationship that they have with their clients and it is especially important to avoid proselytizing for one's own preferred terminology or ascribing status to the use of certain terminologies. 
Policies

Each agency has its own policies and ways of working that have developed for good reasons, based on its primary mandates. These policies extend to every aspect of work, extending across agency boundaries, such as how each deals with confidentiality, keeping of files, who is seen (or treated or counselled), and why they are seen.

Take the example of one program that combined several governmental agencies into one integrated service provider. The most serious problem the new agency had was, paradoxically, the major hindrances to effective communication within the program. Because the various programs continued to exist as self-contained units within the bigger system, they had no formal process with which to share information. Furthermore, the issues around how clients could give formal consent for information to be shared within the program was an ethical nightmare, given the different roles and standards for confidentiality of the ministries of mental health (MH) and social services (MSS) that contributed most of the programs. For example, MSS workers were required to report any legal violations that a teen client made to the caseworker for the teen. Confidentiality requirements dictated that MH staff never did this, unless there was a real danger or unless there was an agreement beforehand that this would be done. Imagine if a therapist treating a teen talked about a violation to a youth worker who then reported it to the caseworker!

The first step was to devise a consent form. It informed clients about who their information would be shared with. It took many drafts and was commented on by everyone in the program-including, importantly, the support staff-before consensus was reached on a document that everyone could use. It allowed staff to be satisfied that they could consult with one another with the informed consent of consumers. Along with the document, several training sessions around issues of confidentiality and consent were conducted, and an ethics committee was created to deal with the many confusing issues that arise in this area.

\section{Politics}

Each profession has its own political agenda (professionalization, money and the like), rules about who can supervise whom, and a sense of its role. What do we each call ourselves: workers, therapists, counsellors, doctors? Each name carries its weight of history and status. How do we refer to ourselves as a group? There are also politics at progressively higher levels. How do we relate to the community agencies with which we work? Can they accept our needs? Can all of us put aside our own needs to work for the good of our clients? At the level of politics, there are many restrictions on what any given agency can do. Alternatively, some areas of funding are more politically correct or acceptable than others, resulting in obvious imbalances in funding services. In one collaborative setting, one team had a very large quantity of attractive supplies that other teams which worked directly with children could not afford. This set the scene for rivalry and resentments between the various teams, all of whom provided much-needed services.

\section{Practicalities}

There is a final $P$ : practicalities. It takes time and training to develop the ability to work with other disciplines. Working together as a team requires that people, at the very least, take time to attend meetings of various kinds, directed either to planning for a specific client or to developing more effective working relationships. When the 
staff is so overworked that finding time to plan for clients is difficult, time to think through solving interprofessional problems is even more limited.

\section{Solutions: The Four $M s$}

There are also four major areas (Ms) in which solutions may be found: make sense out of interactions, meet needs, mend fences, and maintain relationships. These techniques are designed to increase openness to other disciplines and create respectful interactions that are focused on improving communication and decreasing conflict.

I suggest that agencies train their staff in four general types of solutions, the $M \mathrm{~s}$ in the mnemonic. The first is simply to try to make sense out of interactions by determining the underlying needs of the other providers, no matter which $P$ they stem from. The second solution, simple as it sounds, is to try to meet those needs, unless there is a compelling reason to do otherwise. The third solution is to mend fences as quickly as possible after a conflict between staff. The fourth solution is to constantly maintain relationships in order to prevent conflicts from arising.

\section{Make sense out of interactions}

Most providers have developed expertise in understanding the needs of their clients. It can be fairly straightforward to apply that expertise to understanding the needs of our colleagues. For example, one psychotherapist was working with a child from a day care centre. Her agency received a lot of referrals from the centre and it was important to maintain good relations with its director. On the other hand, the director's excessive phone calls, raising concerns about how the therapist was dealing with the child's problems, were difficult to manage. When the therapist reflected on the situation, it became clear that the director was very concerned for the child. She was an active, directing sort of person and wanted to be sure that she knew what was happening in the therapy. On the other hand, she interfered with the therapist's work because agency policies required the therapist to respond to these calls quickly and so they added to her workload and her stress. Although the therapist was extremely professional, the two had created a kind of pursuit-retreat dynamic which left both of them feeling unsatisfied. The therapist decided to offer the director once a week phone calls at a time that was agreeable to both. It would meet the director's need to be informed about her client's progress, the therapist's need to control the calls she received from the director, and, importantly, the need to co-ordinate the work that was being done for the client.

\section{Meet needs}

It is important for providers to develop a certain amount of creativity and flexibility in order to meet the needs of other providers. Sometimes it can be helpful to yield to another's need, even if it conflicts with one's own need. One agency had to find a way to combine staff members from a number of small, independently functioning programs into a supervision group. A lot of consultation was carried out with staff members on how they would like to combine the programs. Most staff members were comfortable meeting as one group, but this did not work for several of them. One person supervised a number of other workers and thus required individual supervision. The other strongly preferred individual supervision and this too was accommodated. Had the second person been forced to attend group supervision, it would probably have led to resentment and lack of co-operation. On the other hand, 
the individual supervision sessions were very productive and well worth adding the extra hour every week.

\section{Mend fences}

It is very important to develop the skills to solve problems as soon as they arise. As a therapist on a team that frequently had to work with social services, I found that other therapists often resorted to solving interprofessional problems by taking them to their own supervisors or to the supervisor of the other professional. This may need to happen, but should be avoided if possible. When supervisors become involved, it can be useful to stop and make explicit the process that led to the conflict. At one point, a colleague complained about me to her supervisor who then complained to my supervisor, who spoke to me. Rather than deal with my colleague through our intermediaries, I phoned her and asked her to explain to me directly what the problem was. She told me that, according the written protocols between our two agencies, I was supposed to give her a regular written summary of the progress of treatment for our mutual client. I had not done that. I acknowledged the validity of her wish to be kept informed about the client and told her that, with my own time pressures, I simply could not write regular reports on all of my clients. Instead, I offered to call her regularly and update her over the phone about the client. We developed a very close working relationship. The intent of the original protocol between the agencies was to deal with ongoing conflicts over management of clients. However, the protocol became unnecessary when I took a problem-solving attitude to working with other agencies.

\section{Maintain relationships}

We need to build simple human relationships, with respect for the kinds of work we each do, the special skills and knowledge each of us brings to our work, a tolerance for the differences amongst us, and a willingness to support each other through the difficult times. This solution directly reflects the important role of positive relationships as emphasized by Seaburn and colleagues (1996). In fact, it is important for all staff to be trained, before conflicts arise, to recognize that developing and maintaining interprofessional relationships is crucial. It can be very useful to hire someone to do conflict resolution training for staff, to teach ways to get unstuck from confrontational positions, to look for underlying commonalities and to seek workable solutions. Such training opportunities should be offered to all the agencies in the community that work together as a cost-effective way of doing "preventive maintenance."

\section{Focusing on Relationships: The $\boldsymbol{R}$}

Some simple "rules of conduct" can go a long way towards maintaining healthy working relationships. First, it is important for all staff to develop a consistent attitude of respect for everyone they work with, both clients and providers. This is much easier said than done, as status and power differences are an integral part of many work settings and cause substantial difficulty. Routinely commenting positively to other providers on the work they do with you (as appropriate) can go a long way to increasing the sense of respect within a group. Recognizing your own strengths and capacities, as well as learning more in areas where you are weak can help you to respect yourself, even in situations where you are experiencing disrespect. Second, it is important for all staff to be trained in good communication skills at a very basic level. Leaving clear, understandable messages is a much rarer art than one would 
guess amongst professionals whose training usually emphasizes communication. One supervisor left an urgent message to call because of an unspecified problem. The therapist was working with severely disturbed children, and became very anxious about the reason for this call. It is often difficult to find a time when two workers are able to reach each other in person and the supervisor's communication didn't indicate when she would be available. The difficulty in responding to the call resulted in substantial stress for the therapist. In the end, the problem was relatively trivial and easily resolvable. The supervisor did not indicate anything about the problem, even though it wasn't confidential. Thus, it was impossible to leave her a message with the answer. When people are overwhelmed, they often communicate quickly, without thinking about how their communication will be received. In times of crisis, it becomes even more important to slow down and communicate in ways that encourage solutions rather than spread anxiety and confusion. E-mails are particularly vulnerable to miscommunication, since they are easy to dash off in emotional moments and easy to misinterpret and share with others.

When staff develop legitimate disagreements over treatment in a case, a focus on maintaining the working relationship can be very helpful. One therapist was working with a young boy who was showing early signs of gender identity disorder, behaving in many ways like a girl. The therapist felt that the child's mother was, in some ways, under the guise of accepting her child's differences, actually reinforcing behaviours that weren't necessarily an integral part of the child's identity. The therapist wanted to encourage the boy to behave in more traditionally male ways. The counsellor at the boy's school strongly disagreed with this position and had, in fact, told the child that he could get a gender change operation when he grew up. In this case, legitimate disagreements about appropriate treatment, reflective of differences in the treatment literature, had resulted in the boy and his family being treated in two completely irreconcilable ways. A telephone consultation with an expert in the field was arranged and the therapist chose to invite the counsellor, maintaining the relationship. As it turned out, the expert suggested following the treatment plan developed by the therapist and all members of the team then followed this plan consistently.

\section{FINAL THOUGHTS}

This brief set of guidelines for working collaboratively is not intended to replace more thorough training; rather, it is intended to help professionals recognize the importance of developing working relationships. The model suggests some easy-to-use ways to implement a collaborative perspective. The literature on collaboration is growing, as is the impetus for collaboration amongst health professionals. Despite substantial progress, existing research is not well integrated and there is no consistent framework that can be used to pull together the disparate elements identified in the literature into an easily teachable set of tools that is so badly needed (Joseph, 2001; Nichols et al., 2002). This framework begins to address the need for such tools, but requires additional development.

This framework has been presented at a number of conferences and participants have indicated that it is helpful. It would be beneficial, now, to test whether use of the framework by individuals or teams helps to promote collaboration. There are a substantial number of instruments recently developed to measure a variety of aspects of team functioning (Heinemann \& Zeiss, 2002b; Heinemann, Schmitt, Farrell, \& Brallier, 1999). The PMR framework is currently being tested by the author as a self- 
instructional, internet-delivered tool for physicians to manage their professional relationships.

How can access to pragmatic, concrete information on interprofessional relationships be ensured? Such access is limited. There are relatively few journals that publish information directed to practitioners rather than researchers. The Enhancing Interdisciplinary Collaboration Project that is currently under development by Health Canada may offer one route to creating usable resources. Contact the EICP secretariat at eicp@conferenceboard.ca for more information.

Another potential route is the Internet. It offers users the opportunity to connect to a host of resources, to modify programs to suit individual users, and to access vivid information. Further, it is easily available, low cost, and easy to use (Rimal \& Flora, 1997). Discussion boards which allow users to share problems and solutions could be particularly useful. Collaboration has been identified as important to the provision of health care in Canada (Romanow, 2002). Thus, creating a central resource on this topic for health care providers across disciplines and across Canada could be of substantial benefit.

Professional organizations such as the Canadian Psychological Association, the Canadian Association of Social Workers, and others could create resources that are made available to practitioners across disciplines through training at conferences, internet postings, and publications. Resources that could be useful include tip sheets, casebooks based on the PMR framework or others, and training videos.

"Power comes not from power everywhere, but from knowing where to put it on” (Maclean, 1976, p. 5). Though Norman Maclean is talking about fly-fishing in this statement, it is equally applicable to interprofessional work. For a variety of reasons, many providers get caught up in power struggles where each tries to maintain control over a situation (Caplan \& Caplan, 1993; Liberman et al., 2001; Sanders et al., 1999; Waxman et al., 1999). Yet often more can be gained by taking what family therapists call a "one-down" position and post-modern therapists characterize as a stance of enquiry or "not-knowing" (Anderson, 1997). I like to think of it as "being Columbo," the bumbling detective who always seems confused about what the other characters are doing. As they explain their acts, Columbo is able to understand the complex relationships of the characters, and ultimately, "puts power on" by suggesting solutions that incorporate those relationships.

There is a story that I like to tell about the importance of working together. A father walks over to his little boy who is struggling to lift a very large rock. The father asks his son if he is using all his strength to lift the rock. The son says he is but the father replies, "You are not using all of your strength because you are not asking me to help." Often those of us working in interdisciplinary settings are not using all our strength and may even be undermining the strength we have by failing to work well with our colleagues.

\section{NOTE}

1. While some authors (Fatout \& Rose, 1995) differentiate between multidisciplinary and interdisciplinary work, and some workers in the field talk about transdisciplinary work, for the purposes of this paper I will use the term multidisciplinary to refer to any work that is carried out jointly by members of different disciplines. 


\section{RÉSUMÉ}

Le travail d'équipe multidisciplinaire est de plus en plus considéré la manière idéale de fournir des services de santé mentale, particulièrement pour les cas impliquant des enfants ou des familles. Cependant, les professionels ont peu d'information pour les aider à reconnaître et surmonter les problèmes associés à ce genre de travail collaboratif. Les ressources transdisciplinaires sont aussi difficiles à obtenir et à utiliser. Le but du présent article est de décrire un système pratique pour aider à l'identification et à la résolution des problèmes associés au travail collaboratif des professionels de santé mentale.

\section{REFERENCES}

Anderson, H. (1997). Conversation, language, and possibilities: A postmodern approach to therapy. New York: BasicBooks.

APA. (1994). Comprehensive and coordinated psychological services for children: A call for service integration. Washington, DC: Author.

Boone, M., Minore, B., Katt, M., \& Kinch, P. (1997). Strength through sharing: Interdisciplinary teamwork in providing health and social services to northern Native communities. Canadian Journal of Community Mental Health, 16(1), 15-29.

Bray, J.H., Enright, M.F., \& Rogers, J. (1997). Collaboration with primary care physicians. In J.A. Morris (Ed.), Practicing psychology in rural settings: Hospital privileges and collaborative care (pp. 55-65). Washington, DC: American Psychological Association.

Caplan, G., \& Caplan, R. (1993). Mental health consultation and collaboration. San Francisco: Jossey-Bass Publishers.

Davis, T. (2001). From pilot to mainstream: Promoting collaboration between mental health and medicine. Families, Systems, and Health, 19(1), 37-45.

Dosser, D.A., Handron, D.S., McCammon, S.L., Powell, J.Y., \& Spencer, S.S. (2001). Challenges and strategies for teaching collaborative interdiciplinary (sic). Families, Systems \& Health, 19(1), 65-82.

Drinka, T., \& Clark, P.G. (2000). Health Care Teamwork. Westport, CT: Auburn House.

Fatout, M., \& Rose, S. (1995). Task groups in the social services. Thousand Oaks, CA: Sage.

Felker, B., Barnes, R., Greenberg, D., Chaney, E., Shores, M., Gillespie-Gately, L., et al. (2004). Preliminary outcomes from an integrated mental health primary care team. Psychiatric Services, 55(4), 442-444.

Freeth, D. (2001). Sustaining interprofessional collaboration. Journal of Interprofessional Care, 15(1), 37-46.

Hackman, J.R. (2004). What makes for a great team. Psychological Science Agenda, 18(6). Retrieved June 14, 2004 from http://www.apa.org/science/psa/june04.html

Haley, W.E., McDaniel, S.H., Bray, J.H., Frank, R.G., Heldring, M., Johnson, S.B., et al. (2004). Psychological practice in primary care settings: Practical tips for clinicians. In R.G. Frank \& S.H. McDaniel (Eds.), Primary care psychology (pp. 95-112). Washington, DC: American Psychological Association.

Hargrove, D.S., \& Keller, P.A. (1997). Collaboration with community mental health centers. In J.A. Morris (Ed.), Practicing psychology in rural settings: Hospital privileges and collaborative care (pp. 67-80). Washington, DC: American Psychological Association.

Harmon, S.K., Braillier, S., \& Brown, G.F. (2002). Organizational and team context. In G.D. Heinemann \& A.M. Zeiss (Eds.), Team performance in health care: Assessment and development (pp. 57-70). New York: Kluwer Academic/Plenum Publishers.

Heinemann, G.D. (2002). Teams in health care settings. In G.D. Heinemann \& A.M. Zeiss (Eds.), Team performance in health care: Assessment and development (pp. 3-17). New York: Kluwer Academic/Plenum Publishers.

Heinemann, G.D., Schmitt, M.H., Farrell, M.P., \& Brallier, S.A. (1999). Development of an attitudes toward health care teams scale. Evaluation \& the Health Professions, 22(1), 123-142.

Hinde, R.A. (1999). Commentary: Aspects of relationships in child development. In W.A. Collins \& B. Laursen (Eds.), Relationships as developmental contexts (pp. 327). Mahwah, 
NJ: Lawrence Erlbaum Associates.

Heinemann, G.D., \& Zeiss, A.M. (2002a). A model of team performance. In G.D. Heinemann \& A.M. Zeiss (Eds.), Team performance in health care: Assessment and development (pp. 2942). New York: Kluwer Academic/Plenum Publishers.

Heinemann, G.D., \& Zeiss, A.M. (2002b). Team performance in health care: Assessment and development. New York: Kluwer Academic/Plenum Publishers.

Johnson, P., Wistow, G., Schulz, R., \& Hardy, B. (2003). Interagency and interprofessional collaboration in community care: The interdependence of structures and values. Journal of Interprofessional Care, 17(1), 69-83.

Joseph, E.T. (2001). Book reviews. Families, Systems, and Health, 19, 101-102.

Lambert, M.T. (2002). Linking mental health and addiction services: A continuity-of-care team model. The Journal of Behavioral Health Services \& Research, 29(4), 433-444.

Lewandowski, C., \& GlenMaye, L. (2002). Teams in child welfare settings: Interprofessional and collaborative processes. Families in Society, 83(3), 245-256.

Liberman, R.P., Hilty, D.M., Drake, R.E., \& Tsang, H.W.H. (2001). Requirements for multidisciplinary teamwork in psychiatric rehabilitation. Psychiatric Services, 52(10), 1331-1342.

Maclean, N. (1976). A river runs through it. Chicago, IL: University of Chicago Press.

McDaniel, S.H., Campbell, T.L., \& Seaburn, D.B. (1990). Family-oriented primary care: A manual for medical providers. New York: Springer-Verlag.

Nichols, L.O., DeFriese, A.M., \& Malone, C.C. (2002). Team process. In G.D. Heinemann \& A.M. Zeiss (Eds.), Team performance in health care: Assessment and development (pp. 7188). New York: Kluwer Academic/Plenum Publishers.

Patterson, J.E. (2001). Training: The missing link in creating collaborative care. Families, Systems, and Health, 19(1), 53-53.

Rimal, R.N., \& Flora, J.A. (1997). Interactive technology attributes in health promotion: Practical and theoretical issues. In R.L.J. Street, W.R. Gold, \& T. Manning (Eds.), Health promotion and interactive technology: Theoretical applications and future directions (pp. 19-38). Mahwah, NJ: Lawrence Erlbaum Associates, Inc.

Romanow, R. (2002). Building on values: The future of health care in Canada.

Ruddy, N.B., \& Schroeder, C.S. (2004). Making it in the real world: Diverse models of collaboration in primary care. In R.G. Frank \& S.H. McDaniel (Eds.), Primary care psychology (pp. 149-168). Washington, DC: American Psychological Association.

Sanders, K., Brockway, J.A., Ellis, B., Cotton, E., \& Bredin, J. (1999). Enhancing mental health climate in hospitals and nursing homes: Collaboration strategies for medical and mental health staff. In M. Duffy (Ed.), Handbook of counseling and psychotherapy with older adults (pp. 335-349). New York: John Wiley \& Sons, Inc.

Seaburn, D.B. (2001). Forward to basics. Families, Systems \& Health, 19(1), 47-51.

Seaburn, D.B., Lorenz, A.D., Gunn, W.B.J., \& Gawinski, B.A. (1996). Models of collaboration: A guide for mental health professionals working with health care practitioners. Basic Books, Inc.

Waxman, R.P., Weist, M.D., \& Benson, D.M. (1999). Toward collaboration in the growing education-mental health interface. Clinical Psychology Review, 19(2), 239-253.

Weist, M.D., Lowie, J.A., Flaherty, L.T., \& Pruitt, D. (2001). Collaboration among the education, mental health, and public health systems to promote youth mental health. Psychiatric Services, 52(10), 1348-1351. 